\title{
Aging and the alimentary tract
}

\section{Biliary tract diseases in the elderly: management and outcomes}

\begin{abstract}
Introduction
Diseases affecting the gall bladder and bile ducts occur commonly in the elderly. By the age of 70 , cholelithiasis, the most frequently occurring disorder affecting these organ systems, and its sequela, choledocholithiasis, are found in $33 \%$ of the population of the United States. ${ }^{1}$ The challenges facing the clinician responsible for treating elderly patients with biliary tract diseases are: (1) developing an astute clinical acumen while assimilating the ubiquitous nature of these disorders in the elderly, especially when compared with the presenting complaints of younger individuals, and (2) acknowledging the increasing number of treatment options now available. In this review, we focus on gallstone diseases, including cholelithiasis, cholecystitis, choledocholithiasis, with and without cholangitis, and malignant biliary strictures, far and away the most common disorders of the biliary tree affecting the aged population.
\end{abstract}

\section{Gallstones and cholecystitis}

Asymptomatic gallstones are a common feature of ageing as time, gall bladder dysfunction and the increasing lithogenicity of bile seem to predispose the gall bladders of the more elderly population to precipitation of supersaturated bile and the concomitant crystallisation of cholesterol or calcium bilirubinate into stone material. While it has been accepted dogma that prophylactic surgery should not be recommended in asymptomatic patients, the longer one has gallstones, the more likely one is to develop cholecystitis and biliary colic. The dilemma clinicians and surgeons encounter is tackling this problem and arriving at the decision on whom to operate and the timing of surgery.

Most patients with gallstones never develop acute cholecystitis, and among those who experience an episode of biliary colic, nearly half will never experience a second episode of colic within five years. Such an outcome could justify a "wait-and-see" approach for most elderly patients. However, there are several factors which contradict such a decision in the aged. Firstly, the presentation of colic in the elderly patient with diabetes and diabetes associated neuropathy is obfuscated and atypical. In such patients, a condition as serious as gangrenous cholecystitis can present with minimal temperature increases, without significant leucocytosis and few, if any, abdominal complaints. ${ }^{2}$ Consequently, clinically significant cholecystitis can be interpreted as another episode of mild biliary colic. The second factor, which is probably more important, is the association of other co-morbid, and, sometimes, life threatening conditions which will frequently discourage even the most enthusiastic surgeon from performing a cholecystectomy.

Elderly patients who require emergency cholecystectomy tend to do poorly when compared with the outcomes of younger patients. The mortality rates for emergency cholecystectomy among the elderly have been reported to range from 6 to $15 \% .^{3}$ In general, these results are similar to the outcomes when gastrointestinal surgery is performed on the elderly. In contrast, when the elderly undergo elective operations, the morbidity and mortality rates are lower and on a par with the operative outcomes for young patients. ${ }^{4}$ As emergency surgery is poorly tolerated by older patients, it is incumbent upon the medical team to assess thoroughly those patients in need of surgery before subjecting them to cholecystectomy. If possible, surgery should be deferred and rescheduled on an elective basis. While delaying surgery may seem to be an incentive or an inducement to increase the number of elective cholecystectomies, one would hope that such a conservative approach would serve as a deterrent to performing "quick fix operations" such as cholecystostomy, which are usually performed when the patient is too sick to withstand definitive surgery but not well enough to ignore. Interestingly, some surgeons are reluctant to perform laparoscopic cholecystectomies in the aged, an observation the authors share as an anecdote. We discourage this attitude as there is no question that minimally invasive surgery is associated with a lower morbidity resulting from such complications as atelectasis, a direct result of decreased pain. We are not aware that operating with a laparoscope is more difficult or dangerous in a 90 year old than open surgery, and we encourage the use of less invasive surgery in our older patients.

In certain clinical situations, we incorporate ERCP techniques alone in treating patients with cholelithiasis. Cholecystitis, which is a consequence of stones impacted in the cystic duct, is such an example. Stones can be dislodged by vigorously injecting contrast material into the cystic duct or by manipulating the obstructing stone with catheters and guidewires. ${ }^{5}$ Transpapillary stents can be placed into the affected gall bladder via the cystic duct, allowing bile and purulent material to drain into the duodenum thus palliating acute cholecystitis. These alternative, endoscopic palliative procedures can be implemented in patients of any age who are considered unfit for surgery, but such techniques are more appropriately used in acutely ill, elderly patients who present with serious co-morbid conditions. $^{6-8}$

\section{Choledocholithiasis}

Common bile duct stones are frequently found in elderly patients who present with concomitant cholecystitis. In the elderly patient, biliary colic is more likely to accompany bile duct stones with or without cholecystitis. In the general population, $5 \%$ of patients presenting with cholecystitis have co-existing bile duct stones. In the elderly, however, this figure rises to $10-20 \% .^{9}$ In addition, in elderly patients who have undergone an emergency cholecystectomy, the incidence of bile duct stones approaches $50 \%$. Choledocholithiasis is a significant problem in the elderly, especially in those patients who present with gall bladder disease, and such a condition should be considered before planning or embarking on surgical treatment. ${ }^{10-12}$ Preoperatively, attempts should be made to demonstrate and corroborate the suspicion of common bile duct stones, thus facilitating appropriate treatment plans.

It is our opinion that the primary approach for treating stone disease of the bile ducts should be endoscopic - that is, ERCP. The success rate for the endoscopic treatment of 
common bile duct stones approaches $98 \%$ in centres of excellence. Ideally, the procedure should be completed before performing a cholecystectomy, thus permitting modification of the surgical approach in the event of endoscopic failure. Very large stones can either be fragmented by using lithotripsy techniques and/or a stent can be inserted to provide decompression. ${ }^{13-16}$ Additionally, oral ursodiol can be given to soften the stones. Stone disease complicated by strictures is treated either in multiple sessions or palliated by providing long term stenting. There are few situations, indeed, when ERCP cannot be offered as a therapeutic modality for treating choledocholithiasis either before or after, or in the absence of cholecystectomy.

The safety and efficacy of ERCP in the very aged deserves comment. In the general population, ERCP is associated with predictable post-treatment complications, the most common being pancreatitis, which occurs in $5-10 \%$ of cases. ${ }^{17}{ }^{18}$ There is also a $1 \%$ risk of bleeding, perforation, and cholangitis associated with the procedure. Interestingly, the elderly tolerate ERCP as well or better than younger patients who undergo the procedure for the treatment of common duct stones and other biliary tract disorders. Furthermore, there was no incident of pancreatitis in our own series of patients 90 years of age or older who underwent therapeutic ERCP. ${ }^{19}$ In this ongoing series, there was an overall complication rate of only $3 \%$, which was lower than that incurred by our general patient population undergoing similar procedures. ${ }^{10}$ In this series, there were no procedure related mortalities, and the length of hospital stay for many of these patients was limited to two or three days. These data contrast strongly with the published surgical reports of common duct explorations in the elderly. The mortality rate incurred among the elderly in surgical series is $4-10 \%$ for elective procedures, but this figure rises to $20 \%$ in emergency operations. ${ }^{11}$

With this information provided for background, we recommend that the clinical approach to evaluating elderly patients be thoughtful and individualised, incorporating minimally invasive procedures such as ERCP and laparoscopic cholecystectomy whenever possible. Although severely ill patients with significant co-morbid illnesses may be treated optimally with palliative procedures such as cholecystostomy or ERCP, the oldest patients are often good candidates for elective cholecystectomy. Surgery for the elderly patient who experiences recurrent colic should not be refused because he or she is considered too old.

\section{Malignant biliary strictures}

As life expectancy increases and the world population is living longer, there is a keener recognition of multi-organ diseases in the elderly in general and liver diseases more specifically. Such is the case of malignant obstructive jaundice resulting from disorders such as pancreatic carcinoma, bile duct carcinoma, gall bladder carcinoma, and ampullary cancer. As with younger patients, the usual presentation of this syndrome in the elderly consists of the insidious development of cholestasis and jaundice. Pain, also, may be an accompanying feature. Pancreatic cancer, the most common cause of malignant biliary obstruction in the USA, accounts for nearly 27000 cases each year, and it has become the fifth leading cause of death from cancer in this country. The incidence of this dreaded disease has increased worldwide, usually presenting in the sixth and subsequent decades of life.

Before embarking on a treatment plan for malignant obstructive jaundice, the clinical team must take into consideration a number of factors, the most important being the extremely low survival and cure rates associated with this disease. The principle objective in treating patients with malignant biliary diseases, presumed to be unresect- able, is to provide palliation of the jaundice. Doing so restores a sense of well-being, avoids early liver failure and encephalopathy due to chronic obstruction, and improves the patient's nutritional and immunological status. ${ }^{20}$ In spite of establishing an early diagnosis, providing optimal treatment and enabling the patient to experience a prompt resolution of cholestasis and jaundice, most affected people succumb to their disease within six months.

Surgical therapy of malignant obstructive jaundice consists of either attempting a curative resection of the tumour or performing a palliative operation. Unfortunately, the surgical cure rate of pancreatic, bile duct, and gall bladder carcinoma is less than $5 \% .{ }^{21}$ In a series reporting better than expected results, resectability of the pancreatic cancer remained below $30 \%{ }^{22}$ in spite of the high selectivity of the cohort group. Thus, caution must be exercised when considering surgical intervention with cure as the objective in elderly patients. Staging techniques should be maximally used in order to select carefully those patients who are likely to have surgically resectable disease. Spiral computed tomography scans using intravenous contrast can accurately evaluate the extent of the tumour and its invasion, especially when three-dimensional reconstruction, computer generated models of the portal vessels is achieved. Endoscopic ultrasound (EUS) is probably the most sensitive diagnostic modality currently available for predicting resectability. ${ }^{23}$ If available, EUS should be incorporated into the diagnostic algorithm for evaluating the mesenteric vasculature.

Palliative surgery is directed towards relieving jaundice by creating a biliary-enteric anastomosis. If duodenal or gastric outlet obstruction is present (usually in $7-14 \%$ of cases $^{22}$ ) a gastrojejunostomy should also be created. In fact, a double bypass should be provided routinely during palliative surgery as $20 \%$ of patients who have not received a gastrojejunostomy will experience gastric outlet obstruction later if they survive up to one year. Although palliative surgery is highly effective in achieving its goal of circumventing the obstruction, we have not witnessed a survival advantage when compared with non-operative techniques of biliary decompression. As gastric outlet obstruction occurs infrequently in patients with malignant obstructive jaundice, the surgical approach to palliation remains an option, not an absolute. However, for most patients, palliative surgery is not necessary. Given the morbidity and 30 day mortality (up to $20 \%$ ) for a bypass procedure, non-operative techniques of palliation are preferred in an aged population. ${ }^{20}$

Endoscopic relief of jaundice has been used successfully for over 20 years, and these endoscopic techniques have a high rate of success (up to $90-95 \%$ of attempts). ${ }^{24}$ Plastic stents as large in diameter as 12 French can be advanced across the papilla through the area of obstruction within the proximal biliary tree. Greater success is achieved when treating distal bile duct obstruction as cholangitis and other complications occur less frequently in this subset of patients. With tumours affecting the bifurcation of the hepatic ducts (Klatskin), stents can be placed into both the right and left intrahepatic ducts to provide decompression. The endoscopic procedures require about an hour to complete, and most patients spend no more than 36 hours in hospital. After placing biliary stents, the jaundice dissipates almost immediately, unless there is co-morbid liver disease, and, usually, the patient is without pain or ileus after the procedure.

Plastic stents placed endoscopically have a propensity to clog or occlude within six months of placement, thus requiring stent exchange. While large diameter plastic stents survive or function longer than small diameter stents, there is very little one can do to (a) retard the 
accumulation of biofilm within the stent's lumen and (b) prolong its patency. The newest strategy devised to tackle the clogging issue is to insert large diameter, expandable, mesh stents. These metallic endosprostheses seem to delay stent occlusion for up to six months, longer than that expected or witnessed with plastic stents. ${ }^{25}$ However, the expandable metal stents are (a) much more expensive than the plastic stents, and $(b)$ are not removable. After stent insertion, most patients die in an anicteric state before the metal stents occlude, and their use is a reasonable approach to avoid repeat hospital visits and endoscopies in patients who are not expected to survive more than 4-6 months. Frequent return visits to a hospital are costly and remind patients of their mortality. In patients who are expected to survive a year or more, a surgical bypass is recommended in order to avoid the frequent visits necessary to exchange plastic prostheses. However, the elderly rarely survive more than four months, and their stents are usually patent at the time of death.

As the prognosis for patients with malignant biliary obstruction is poor, non-operative palliation is appropriate for most patients, especially elderly patients. The elderly tolerate surgery less well, and spend too many of their remaining days convalescing from their surgery. Therefore, endoscopic stenting is recommended for the vast majority of patients affected by malignant biliary obstruction. Surgical intervention is recommended for those patients whose disease appears to be localised, are very healthy, or in those in whom gastric outlet obstruction is present. "Exploratory" surgery should be avoided.

\section{Summary}

Elderly people commonly present with biliary tract disease. Gallstone disease is an important cause of recurrent abdominal symptoms, and we advocate an aggressive approach in stable patients not at risk to improve the quality of their lives. Choledocholithiasis is optimally treated by ERCP (98\% success) even in patients who are at great risk. Endoscopic intervention often obviates the need for emergency biliary tract surgery in the elderly, is better tolerated, and is associated with significantly less risk and a lower mortality. In contrast, emergency surgery in the elderly is poorly tolerated. Even cholecystitis and biliary pancreatitis (not discussed here) are amenable to endoscopic treatment.

Malignant biliary obstruction should not and cannot be treated as aggressively as benign disorders affecting the biliary tree as the long term outlook is poor. Endoscopic palliation usually suffices in maximising treatment and improving the patient's quality of life with few associated complications or postprocedural machinations (drainage bags or tubes). The afflicted population in general and the elderly in particular benefit from minimally invasive endoscopic decompression techniques.

JEROME H SIEGEL FRANKLIN E KASMIN

Endoscopy Section,

Beth Israel Medical Center North Division,

New York, New York 10128, USA

1 McSherry CK, Ferstenberg H, Calhoun WF, et al. The natural history of diagnosed gallstone disease in symptomatic and symptomatic patients. Ann Surg 1985; 202: 59

2 Cobden I, Venables CW, Lendrum R, James OFW. Gallstones presenting as mental and physical debility in the elderly. Lancet 1984; i: 1062.

3 Glenn F, Hays DM. The age factor in the mortality rate of patients undergoing surgery of the biliary tract. Surg Gynecol Obstet 1955; 100: 11.

4 Glenn F. Surgical management of acute cholecystitis in patients 65 years of age and older. Ann Surg 1981; 193: 56.

5 Siegel JH, Kasmin FE, Cohen SA. Endoscopic retrograde cholangiopancreatography treatment of cholecystitis: possible? yes; practicle?? Diagn Ther Endosc 1994; 1: 51

6 Hosking MP, Warmer MA, Lodbell CM, et al. Outcomes of surgery in patients 90 years of age and older. FAMA 1989; 261: 1909.

7 Glenn F. Cholecystectomy in the high risk patient with biliary tract disease. Ann Surg 1997; 185: 185.

8 Margiotta SJ Jr, Willis IH, Wallack MK. Cholecystectomy in the elderly. Am Surgeon 1988; 54: 34

9 Beaton HL. Surgical considerations. In: Gelb A, ed. Clinical gastroenterology in the elderly. New York: Marcel Dekker, 1996: 271-82.

10 Pigott JP, Williams GB. Cholecystectomy in the elderly. Am f Surg 1988; 155: 408 .

11 Harness JK, Strodel WE, Talsma SE. Symptomatic biliary disease in the elderly patient. Am Surgeon 1986; 52: 442.

12 Krarup T, Sonderstrup J, Kruse-Blinkenberg HO, Schmidt A. Surgery for gallstones in old age: Do we operate too late? Acta Chir Scand 1982; 148: 263 .

13 Siegel JH, Yatto RP. Biliary endoprostheses for the management of retained common bile duct stones. Am f Gastroenterol 1984; 79: 50

14 Siegel JH, Ben-Zvi JS, Pullano WE. Mechanical lithotripsy of common duct stones. Gastrointest Endosc 1990; 36: 351-6.

15 Siegel JH, Ben-Zvi JS, Pullano WE. Endoscopic electrohydraulic lithotripsy. Gastrointest Endosc 1990; 36: 134-6.

16 Siegel JH. Endoscopic retrograde cholangiopancreatography: Technique, diagnosis and therapy. New York: Raven Press, 1992

17 Cotton PB, Chapman M, Whiteside CG, et al. Duodenoscopic papillotomy and gallstone removal. Br F Surg 1976; 63: 709-14.

18 Davidson BR, Neoptolemos JP, Carr-Locke DL. Endoscopic sphincterotomy for common bile duct calculi in patients with gall bladder in situ considered unfit for surgery. Gut 1988; 29: 114-20.

19 Kasmin FE, Fenig DM, Cohen SA, Siegel JH. Biliary endoscopy in nonagenarians- "ERCP in the nineties" [abstract]. Gastrointest Endosc 1995; 41: A423.

20 Siegel JH, Snady H. The significance of endoscopically placed prostheses in the management of biliary obstruction due to carcinoma of the pancreas: results of non-operative decompression in 277 patients. Am $\mathcal{f}$ Gastroenterol 1986; 81: 634-41.

21 Parker SL, Tong T, Bolden S, Wingo PA. Cancer Statistics, 1997. CA Cancer f Clin 1997; 47: 5-27.

22 Gudjonsson B. Editorial: Pancreatic cancer. The need for critical assessment. F Clin Gastroenterol 1996; 23: 2-6.

23 Rosch T. Staging of pancreatic cancer: analysis of literature results. Gastrointest Endosc Clin North Am 1995; 5: 735-40

24 Smith AC, Dowsett JF, Hatfield ARW, Cotton PB. Randomised trial of endoscopic stenting versus surgical bypass in malignant low bile duct obstruction. Lancet 1994; 344: 1655-60.

25 Huibregtse K, Cheung J, Coene PPLO, et al. Endoscopic placement of expandable metal stents for biliary strictures-a preliminary experience with 33 patients. Endoscopy 1989; 21: 280-2. 